See Article page $\mathrm{XXX}$.

\section{Commentary: Finding the way through the maze}

\author{
Arnaldo Dimagli, MD, and \\ Vito Domenico Bruno, MD, PhD
}

Persistent preoperative atrial fibrillation (AF) has been demonstrated to negatively influence the short- and longterm outcomes for patients undergoing cardiac surgery when left untreated. ${ }^{1}$ Concomitant surgical treatment of AF is possible through surgical ablation. The maze technique was performed for the first time 34 years ago by Dr James $\operatorname{Cox}^{2}$ and since then, a growing bulk of evidence has been building that demonstrates optimal long-term rates of sinus rhythm restoration and improved survival in patients undergoing concomitant ablation. ${ }^{3}$ Most of the evidence on the maze procedure comes from mitral valve surgery studies because the proportion of patients with AF is as high as $40 \%$ when degenerative mitral regurgitation is present. ${ }^{4}$ Conversely, the maze procedure combined with tricuspid valve surgery has rarely been under the spotlight.

Park and colleagues ${ }^{5}$ present the long-term outcomes of patients with persistent AF and severe tricuspid regurgitation undergoing the maze procedure. Using propensity score matching, the authors show a more favorable sinus rhythm restoration and a lower rate of major adverse cardiovascular events at 9 years in patients undergoing concomitant maze cryoablation. This report adds to the literature important findings for a specific subgroup of patients. Tricuspid regurgitation usually coexists with biatrial dilatation, which has been shown to be a potential indicator of sinus rhythm restoration failure. ${ }^{6,7}$ However, patients with left and right atrial diameter within a certain limit-60 and $54 \mathrm{~mm}$ in this study - could still benefit from AF ablation, thus preventing exposure to the potential adverse events from $\mathrm{AF}$ and the required anticoagulation. ${ }^{5}$ These

\footnotetext{
From the Bristol Heart Institute, University of Bristol, Bristol, United Kingdom. Disclosures: The authors reported no conflicts of interest.

The Journal policy requires editors and reviewers to disclose conflicts of interest and to decline handling or reviewing manuscripts for which they may have a conflict of interest. The editors and reviewers of this article have no conflicts of interest.

Received for publication Dec 6, 2021; revisions received Dec 6, 2021; accepted for publication Dec 7, 2021.

Address for reprints: Arnaldo Dimagli, MD, Bristol Heart Institute, University of Bristol, Upper Maudlin St, Bristol BS28HW, United Kingdom (E-mail: arnaldo, dimagli@bristol.ac.uk).

J Thorac Cardiovasc Surg 2021; $\mathbf{\square}: 1-2$

$0022-5223 / \$ 36.00$

Copyright (c) 2021 by The American Association for Thoracic Surgery

https://doi.org/10.1016/j.jtcvs.2021.12.014
}

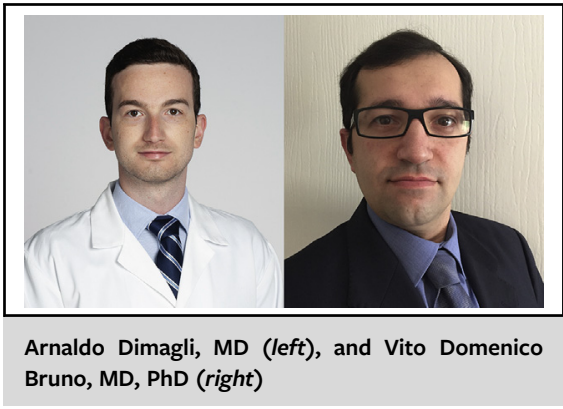

CENTRAL MESSAGE

Patients with severe tricuspid regurgitation may achieve acceptable rates of sinus rhythm restoration after surgical ablation of atrial fibrillation.

findings, although they must be confirmed in further studies, allow us to better define who are the patients who should undergo a maze procedure.

Another relevant concern when treating patients with severe tricuspid regurgitation is related to prolonging the cardiopulmonary bypass time due to surgical ablation. These patients could present with severe sequelae of the chronic valvopathy such as multiorgan failure, and therefore longer operative times could be detrimental. However, Park and colleagues $^{5}$ demonstrate comparable cardiopulmonary bypass times: Just over 2.5 hours for both groups. This article adds to the existing evidence already proving the safety and effectiveness of the maze procedure. Indeed, anticipated longer operative times are often feared by surgeons for the potential association with worst postoperative outcomes and induce surgeons to omit concomitant ablation when it would actually be beneficial.

It is important to note that the article by Park and colleagues ${ }^{5}$ is based on a retrospective analysis of surgical cases. Despite that the authors adopted a sophisticated adjustment methodology based on propensity score matching, it is still possible that residual confounders could have biased the results. In fact, among the limitations of the calculation of propensity score is that it can be computed only on the observed, measured variables (eg, age, sex, and diabetes status), but it does not take into account those patient characteristics that are not measured (eg, fragility). These variables are usually assessed by the eyeball testthat is, surgeon's intuition—and together with the measured variables are weighted in decision-making process. For this 
reason, even a perfectly conducted propensity score matching could still be potentially undermined by selection bias. ${ }^{8}$

The results presented by Park and colleagues ${ }^{5}$ should stimulate wider adoption of concomitant surgical ablation procedures in patients undergoing tricuspid valve surgery for severe tricuspid regurgitation in view of the promising outcomes and rhythm restoration. Particular attention should be paid to optimal patient selection, in particular with regard to the presence of biatrial enlargement that could jeopardize the benefits associated with AF ablation.

\section{References}

1. Saxena A, Virk SA, Bowman S, Bannon PG. Systematic review and meta-analysis on the impact of preoperative atrial fibrillation on short- and long-term outcomes after aortic valve replacement. J Cardiovasc Surg (Torino). 2017;58:943-50.

2. Cox JL. The first maze procedure. J Thorac Cardiovasc Surg. 2011;141:1093-7. https://doi.org/10.1016/j.jtcvs.2010.12.012
3. Otto CM, Nishimura RA, Bonow RO, Carabello BA, Erwin JP III, Gentile F, et al. 2020 ACC/AHA guideline for the management of patients with valvular heart disease: executive summary: a report of the American College of Cardiology/American Heart Association Joint Committee on clinical practice guidelines. Circulation. 2021;143:e35-71.

4. Grigioni F, Benfari G, Vanoverschelde J-L, Tribouilloy C, Avierinos J-F, Bursi F, et al. Long-term implications of atrial fibrillation in patients with degenerative mitral regurgitation. J Am Coll Cardiol. 2019;73:264-74.

5. Park I, Jeong DS, Park S-J, Ahn JH, Kim J, Kim EK, et al. Impact of maze procedure in patients with severe tricuspid regurgitation and persistent atrial fibrillation. $J$ Thorac Cardiovasc Surg. 2022. XXX:XXX.

6. Gillinov AM, Gelijns AC, Parides MK, DeRose JJ, Moskowitz AJ, Voisine P, et al. Surgical ablation of atrial fibrillation during mitral-valve surgery. $N$ Engl J Med. 2015;372:1399-409. https://doi.org/10.1056/NEJMoa1500528

7. McClure GR, Belley-Cote EP, Jaffer IH, Dvirnik N, An KR, Fortin G, et al. Surgical ablation of atrial fibrillation: a systematic review and meta-analysis of randomized controlled trials. Europace. 2018;20:1442-50.

8. Gaudino M, Di Franco A, Rahouma M, Tam DY, Iannaccone M, Deb S, et al. Unmeasured confounders in observational studies comparing bilateral versus single internal thoracic artery for coronary artery bypass grafting: a meta-analysis. J Am Heart Assoc. 2018;7:e008010. https://doi.org/10.1161/ JAHA.117.008010 